\title{
Síndrome do túnel carpal: resultados do tratamento cirúrgico com a técnica de mini-incisão
}

\section{Carpal tunnel syndrome: results of surgical treatment with mini-incision technique}

Walter Yoshinori Fukushima', Gustavo Mantovani Ruggiero', Álvaro Baik Cho', Márcio Aurélio Aita', Yussef Ali Abdouni', Henrique Pellacani Fernandes Soutello', Thiago da Motta Mattar', Felipe Galvão Álvares de Abreu'

\begin{abstract}
Resumo
Introdução: A síndrome do túnel carpal é constituída por um conjunto de sinais e sintomas característicos como parestesia, formigamento, dor e perda da força nas mãos. Inicialmente os sintomas são brandos, progredindo ao longo do tempo. Objetivo: Apresentar os resultados do tratamento cirúrgico com mini-incisão, bem como discutir a relação entre os resultados e a técnica cirúrgica utilizada. Método: Esta pesquisa foi realizada na Faculdade de Medicina do $A B C$, onde foram avaliados 79 punhos de 71 pacientes, todos submetidos ao tratamento cirúrgico da síndrome do túnel carpal por "miniincisão", entre janeiro de 1996 e maio de 2007. A média de idade entre os pacientes foi de 52,4 anos, sendo a idade mínima 27 e a máxima 80 anos. Sessenta e cinco pacientes eram do sexo feminino e seis do masculino. Quanto ao lado acometido, foram obtidos 36 punhos do lado direito e 27 do lado esquerdo, sendo 8 bilaterais. Resultados: 0s resultados clínicos mostraram que 60 pacientes ficaram satisfeitos com os sintomas, com os resultados estéticos e funcionais e com a melhora da força muscular para apreensão. Dois pacientes apresentaram complicações cutâneas com infecção superficial e necessitaram de antibioticoterapia oral. Conclusões: Concluímos que o tratamento cirúrgico por "mini-incisão" é uma técnica segura, com poucas complicações e resultados clínicos e estéticos satisfatórios para o tratamento da síndrome do túnel do carpo.
\end{abstract}

Palavras-chave: Síndrome do túnel carpal; dor; parestesia; punho.

\section{Abstract}

Introduction: The carpal tunnel syndrome is composed of signs and characteristic symptoms as parestesis, tingling, pain and hand power loose. Initially, the symptoms appear softly, progressing along the time. Objective: To present the results of surgical treatment using mini-incision, as well as to discuss the relationship between the results and the surgical technique used. Method: This study was accomplished at Faculdade de Medicina do ABC where 79 wrists of 71 patients were evaluated. All of them were submitted to the surgical treatment of the carpal tunnel syndrome using "mini-open" technique between January 1996 and May 2007. The mean age of the patients was 52.4 years old, the minimum age was 27 years old and the maximum was 80 years old. Sixty-five patients were females and six were males. Thirty-six subjects had the right side affected and 27 had the left side, though 8 patients presented both sides affected. Results: The clinical results showed that 60 patients were satisfied with the symptomatic, aesthetic and functional results, as well as with the muscular power improvement for apprehension. Two patients presented cutaneous complications with superficial infection and were treated with oral antibiotic therapy. Conclusions: The surgical treatment using "mini-incision" is a safe technique, with few complications and satisfactory clinical and aesthetic results for the carpal tunnel syndrome treatment.

Keywords: Carpal tunnel syndrome; pain; paresthesia; wrist.

Recebido: $19 / 5 / 2009$

Revisado: 26/9/ 2009

Aprovado: $28 / 12 / 2009$

Trabalho realizado no Hospital Estadual Mário Covas, Fundação do ABC, Santo André (SP), Brasil

Disciplina de Ortopedia e Traumatologia da Faculdade de Medicina do ABC (FMABC), Santo André (SP), Brasil

Endereço para correspondência: Walter Yoshinori Fukushima - Rua das Aroeiras, 191 - Bairro Jardim - CEP 09090-000 - Santo André (SP) -

e-mail:walterfukushima@ig.com.br 


\section{Introdução}

A síndrome do túnel carpal representa a neuropatia compressiva mais comum, melhor definida e mais estudada do ser humano. A compressão do nervo mediano no túnel carpal decorre, na maioria dos casos, de tenossinovite crônica flexora não específica, podendo ocorrer em muitas outras doenças ou lesões que ocupem espaço no túnel do carpo ${ }^{1}$.

Suas principais manifestações clínicas são dor, parestesia, dormência, perda de força e formigamento das mãos. No início, a sintomatologia é leve; com o tempo, ocorre piora do quadro, com diminuição da sensibilidade e da força muscular, atrofia e o despertar noturno ${ }^{2,3}$.

O diagnóstico clínico e eletrofisiológico precisos conduzem à cura completa na maioria dos casos ${ }^{4,5}$. Entretanto, esse diagnóstico é tardio, e a descompressão cirúrgica do túnel do carpo é o procedimento mais frequentemente realizado pelo cirurgião da mão; não existe, no entanto, um consenso sob a melhor técnica a ser empregada ${ }^{6,7}$.

Dessa maneira, o objetivo é descrever os resultados cirúrgicos da técnica de mini-incisão na síndrome do túnel carpal e a prevalência de sexo e membro dominante.

\section{Método}

$\mathrm{O}$ estudo foi realizado na Faculdade de Medicina do ABC (FMABC) no período de janeiro de 1996 e maio de 2007, com 71 pacientes e 79 punhos, atendidos nos ambulatórios mediante termo de consentimento livre e esclarecido e protocolo de atendimento e de acompanhamento pós-operatório.

Os critérios de inclusão foram: adultos de ambos os sexos com diagnóstico clínico e eletroneuromiografia compatível com a síndrome do túnel carpal. Os critérios de exclusão foram os pacientes com outra doença neurológica comprovada e operações anteriores. Todos os pacientes foram submetidos ao tratamento cirúrgico por meio da técnica de mini-incisão que consiste na técnica aberta com incisão de $3 \mathrm{~cm}$ na face palmar na região do túnel carpal (Figura 1). Os cuidados pós-operatórios consistiram no segmento ambulatorial com curativos semanais e retirada dos pontos após duas semanas. Observamos a melhora do complexo sintomático (dor, parestesia e dormência das mãos), o aspecto da ferida operatória e o restabelecimento da função do membro. Esta pesquisa foi aprovada pelo Comitê de Ética da FMABC, parecer 129/2009.

\section{Resultados}

A faixa etária dos indivíduos variou entre 27 e 80 anos. Sessenta e cinco pacientes eram do sexo feminino e seis do sexo masculino. Quanto ao lado acometido, foram obtidos 36 punhos do lado direito e 27 do lado esquerdo, sendo 8 bilaterais. Os resultados estéticos foram satisfatórios $(97,18 \%)$ e quanto aos resultados clínicos, 60 pacientes ficaram satisfeitos (84,5\%), dois deles apresentaram complicações cutâneas com infecção superficial e necessitaram de antibioticoterapia oral.

\section{Discussão}

Os achados clínicos e epidemiológicos revelaram ampla predominância da síndrome do túnel carpal no sexo feminino (65 casos), valor próximo ao da literatura brasileira e aproximadamente $75 \%$ mai elevado em relação à literatura internacional. O estudo pode demonstrar como é incomum a existência dessa patologia no sexo masculino, o que pode nos levar a acreditar no papel importante que tem os hormônios femininos nessa afecção. O horário mais comum do quadro álgico foi noturno-matinal (60 casos), quadro característico da compressão carpal.

A dor inespecífica relacionada à compressão nervosa, diferente da dormência e parestesia, pode ocorrer abaixo (40 casos) ou acima do bloqueio do carpo (31 casos), como no antebraço, braço e ombro. O lado sintomático referido correspondeu a 8 casos bilaterais, 36 casos do lado direito e 27 casos do esquerdo. A faixa etária era bastante ampla, sendo o mais jovem com 27 anos e o mais idoso com 80 anos $^{8-10}$.

Pacientes gestantes podem desenvolver quadro de síndrome do túnel carpal e as manifestações podem ser mais evidentes no final da gravidez e devem ser diferenciadas das chamadas idiopáticas, inclusive no tratamento ${ }^{11}$.

Os resultados obtidos neste estudo podem ser comparados a outros. As prevalências maiores em mulheres são notórias, com predominância para o lado direito e relação direta com os aspectos trabalhistas, necessidade de afastamento do trabalho ou readaptação de cargo e/ou indenização ${ }^{9,10,12}$.

Em relação ao tratamento, inúmeras técnicas cirúrgicas foram descritas e, sendo assim, não existe um consenso da melhor técnica utilizada. A técnica escolhida tem a vantagem de propiciar ao paciente um excelente resultado estético-funcional, proporcionando uma exploração detalhada do túnel carpal e não somente a liberação do ligamento transverso do carpo, como descrito em procedimento endoscópico. Ob-

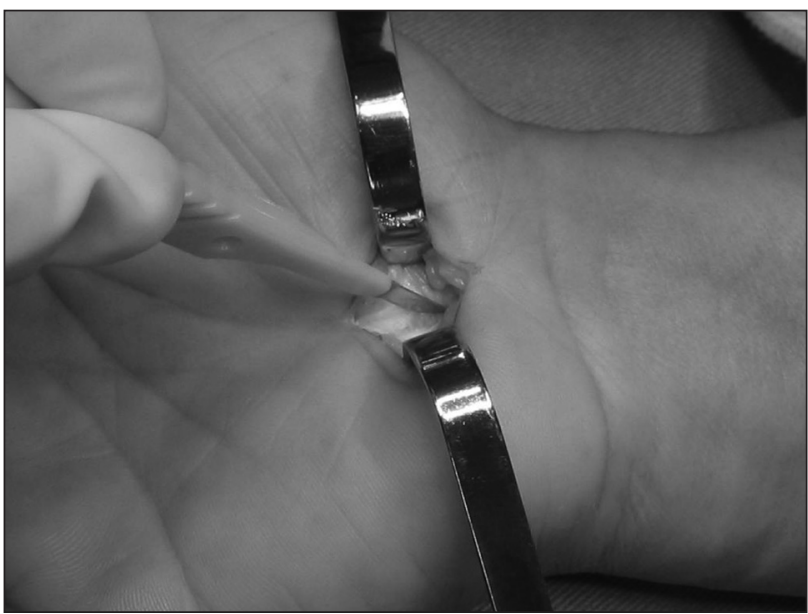

Figura 1 - Aspecto da mini-incisão no punho com início da abertura do túnel carpal. 
servou-se a melhora da sintomatologia em 60 casos e apenas 2 pacientes apresentaram infecção superficial da pele, os quais foram tratados com antibioticoterapia oral, com total remissão do processo infeccioso.
A técnica cirúrgica da mini-incisão demonstra ser segura, eficaz e atraumática no tratamento da síndrome do túnel carpal. Apresenta prevalência de utilização no sexo feminino e no lado dominante.

\section{Referências}

1. Brain WR, Wright AD, Wilkinson M. Spontaneous compression of both median nerves in the carpal tunnel: six cases treated surgically. Lancet. 1947;1(8):277-82.

2. Phalen GS. Spontaneous compression of the median nerve at the wrist. J Am Med Assoc. 1951;145(15):1128-33.

3. Phalen GS. The carpal-tunnel syndrome. Seventeen years' experience in diagnosis and treatment of six hundred fifty-four hands. J Bone Joint Surg Am. 1966;48(2):211-28.

4. You H, Simmons Z, Freivalds A, Kothari MJ, Naidu SH. Relationships between clinical symptom severity scales and nerve conduction measures in carpal tunnel syndrome. Muscle Nerve. 1999;22(4):497-501.

5. Gerr F, Letz R. The sensitivity and specificity of tests for carpal tunnel syndrome vary with the comparison subjects. J Hand Surg Br. 1998;23(2):151-5.

6. Katz JN, Larson MG, Sabra A, Kraup C, Stirrat CR, Sethi R, et al. The carpal tunnel syndrome: diagnostic utility of the history and physical examination findings. Ann Intern Med. 1990;112(5):321-7.
7. Nathan PA, Meadows KD, Doyle SL. Relationship of age and sex to sensory conduction of the median nerve at the carpal tunnel and association of slowed conduction with symptoms. Muscle Nerve. 1988;11(11):1149-53.

8. Kouyoumdjian JA. Síndrome do túnel do carpo, aspectos clínicoepidemiológicos em 668 casos. Arq Neuropsiquiatr. 1999;57(2A):202-7.

9. Hadler NM. Repetitive upper-extremity motions in the workplace are not hazardous. J Hand Surg Am. 1997;22(1):19-29.

10. Vender MI, Kasdan ML, Truppa KL. Upper extremity disorders: A literature review to determine work-relatedness. J Hand Surg Am. 1995; 20(4): 534-41.

11. Bradley MP, Hayes EP, Weiss APC, Akelman E. A prospective study of outcome following mini-open carpal tunnel release. Hand Surg. 2003; 8(1):59-63.

12. Finsen V, Zeitlmann H. Carpal tunnel syndrome during pregnancy. Scand J Plast Reconstr Surg Hand Surg. 2006;40(1):41-5. 